\title{
Intrahepatic Cholestasis of Pregnancy is Associated with Gestational Diabetes Mellitus
}

\author{
Gulenay GENCOSMANOGLU TURKMEN', Zehra VURAL YILMAZ¹, Yuksel OGUZ¹, Kadriye YAKUT ${ }^{1}$ \\ Cem Yasar SANHAL ${ }^{1}$, Dilek UYGUR ${ }^{1}$
}

Ankara, Turkey

\begin{abstract}
OBJECTIVES: Intrahepatic (also known as obstetric) cholestasis of pregnancy is one of the most frequently diagnosed conditions for pregnancy-specific hepatic disease. It has consistently been found to be related to adverse pregnancy outcomes. In recent studies, a relationship between Intrahepatic cholestasis of pregnancy and gestational diabetes mellitus was demonstrated. However, the association between serum total total bile acid evel in Intrahepatic cholestasis of pregnancy and gestational diabetes mellitus is not fully understood. This study aims to evaluate the association between serum total total bile acid levels in Intrahepatic cholestasis of pregnancy disease with gestational diabetes mellitus.
\end{abstract}

STUDY DESIGN: Eighty pregnant women diagnosed with Intrahepatic cholestasis of pregnancy and eighty healthy pregnant women as normal controls were included in the study. Their clinical characteristics and laboratory test results including liver function tests, glucose challenge tests, glucose tolerance tests, and fasting and postprandial total total bile acid levels were recorded. Cases with serumtotal total bile acid levels between 12-40 $\mu \mathrm{mol} / L$ were described as mild disease, $>40 \mu \mathrm{mol} / \mathrm{L}$ was described as severe disease.

RESULTS: The mean $50-\mathrm{g}$ glucose challenge tests value was significantly higher in pregnant women with Intrahepatic cholestasis of pregnancy compared to the healthy controls $(128.7 \pm 28.2,106.6 \pm 27.0$; $p<0.0001)$ and it was slightly higher in women with severe disease than women with mild disease $(132.7 \pm 30.1,125.5 \pm 26.5 ; p=0.26)$. The percent of gestational diabetes mellitus diagnosis with Intrahepatic cholestasis of pregnancy disease (11.25\%) was higher than in healthy pregnant women $(6.25 \%)$ but the difference was not found to be statistically significant $(p=0.187)$ and it was similar in pregnant women with mild and severe disease $(11.1 \%, 11.4 \% ; p=0.31)$.

CONCLUSION: Our current study demonstrated that Intrahepatic cholestasis of pregnancy was not associated with gestational diabetes mellitus, also, we did not demonstrate a relationship of total total bile acid level with Intrahepatic cholestasis of pregnancy and gestational diabetes mellitus. It may be due to our study's small sample size. Further and well-designed studies with larger sample sizes are necessary to determine the relationship between gestational diabetes mellitus and Intrahepatic cholestasis of pregnancy and also the function of total total bile acid in the pathogenesis of gestational diabetes mellitus disease.

Keywords: Bile acid, Gestational diabetes mellitus, Intrahepatic cholestasis of pregnancy

Gynecol Obstet Reprod Med 2019;25(3):133-137

${ }^{I}$ University of Health Sciences, Zekai Tahir Burak Women's Health, Education and Research Hospital, Ankara, Turkey

Address of Correspondence: Gulenay Gencosmanoglu Turkmen University of Health Science Zekai Tahir Burak Women's Health, Education and Research Hospital, 06230Ankara, Turkey drgulenay@yahoo.com

Submitted for : Publication Revised for: Publication Accepted for Publication: ORCID IDs of the authors: ZVY: 0000-0002-8719-1879, KY: 0000-0003-3182-4312, $D U: 0000-0001-8567-9048$

\begin{tabular}{|c|c|}
\hline \multirow{3}{*}{ 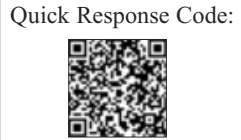 } & Access this article online \\
\hline & $\begin{array}{l}\text { Website: www.gorm.com.tr } \\
\text { e- mail: info@gorm.com.tr }\end{array}$ \\
\hline & DOI:10.21613/GORM.2018.855 \\
\hline
\end{tabular}

\section{Introduction}

Intrahepatic (or obstetric) cholestasis of pregnancy (ICP) is the leading cause of pregnancy-specific hepatic disorder. The incidence of ICP varies markedly among several reports, from as high as $5 \%$ in Chile to $0.7 \%$ in the UK (1). ICP is diagnosed with the elevation of serum aminotransferase concentration and/or elevation of fasting serum total bile acid (TBA) level accompanying pruritus. Pruritus, in particular, becomes symptomatic after the late second trimester of pregnancy and, itching worsens at night, influencing especially the palmar and plantar parts of the hands and feet (2). It resolves spontaneously approximately by four weeks postpartum.

How to cite this article: Gencosmanoglu Turkmen G. Vural Yilmaz Z. Oguz Y. Yakut K. Sanhal CY. Uygur K. Intrahepatic Cholestasis of Pregnancy is Associated with Gestational Diabetes Mellitus. Gynecol Obstet Reprod Med. 2019;25(3):133-137 
There are no factors that are well established in the etiopathogenesis of ICP. Genetic, hormonal, and environmental factors seem likely to play a role in the etiology of the ICP. Inadequate TBA homeostasis in the presence of physiological gestational alterations has been suspected as a reason for ICP (3).

Intrahepatic cholestasis of pregnancy has consistently been related to poor pregnancy outcomes-such as a higher rate of preterm birth, neonatal mortality, small for gestational age at birth and unexplained stillbirth $(2,4)$. The risk of adverse pregnancy outcome is higher if fasting TBA concentration exceeds $40 \mu \mathrm{mol} / \mathrm{L}(5,6)$. In recent studies, it has also been demonstrated that ICP is related to gestational diabetes mellitus (GDM) $(3,7)$, but to the best of our knowledge no one has investigated the association between TBA levels and GDM disease.

From the previous studies, we hypothesized the fact that ICP could have an association with an increased incidence of GDM as a result of the impaired bile homeostasis. In this study, we aimed to examine the relationship between serum TBA levels in ICP and GDM.

\section{Material and Method}

This case-control study was conducted between September 2015 and July 2016 at Zekai Tahir Burak Women's Health Education and Research Hospital, Ankara, Turkey. Data were collected from patients' hospital records. This study was approved by our hospital ethics committee (approval number: 37) and the universal principles of the Helsinki Declaration were applied.

The hospital records of eighty consecutive pregnant women diagnosed with ICP were reached and 80 healthy pregnant women were included as a control group in the study. The two groups of pregnant women were matched according to the maternal age and body mass index (BMI). Pregnant women who had multiple pregnancies, drug use except for vitamins, any disease or viral infection that affected the liver and also those with a skin disease were not included in the study.

Diagnosis of ICP was made if a pregnant woman had generalized new onset persistent pruritus without skin lesions and laboratory results of TBAs exceeding $12 \mu \mathrm{mol} / \mathrm{L}$ and/or elevated liver enzymes in the absence of any identifiable liver disease. ICP group was also divided into two groups as mild and severe depending on the serum TBA levels. Diagnosis of severe disease was made with the serum levels of $\geq 40$ $\mathrm{mmol} / \mathrm{L}(1)$. All the pregnant women in the ICP group were hospitalized and tested for complete blood count (CBC), hepatic enzymes, and serology for hepatitis A, B and C virus for initial evaluation. All women who had ICP were evaluated with hepatic ultrasound examination to exclude other forms of hepatic disease. According to the American College of Obstetricians \& Gynecologists guidelines, a 50 g oral glucose challenge test (50-g GCT) was applied to all participants be- tween 24-28 weeks of gestation in both groups and blood glucose levels were measured 1 hour later. If the blood glucose level was above $140 \mathrm{mg} / \mathrm{dL}$, a $100 \mathrm{~g} 3$-h glucose tolerance test (GTT) was applied, diagnosis of GDM was confirmed if two of the blood glucose test results were above the following levels: fasting $95 \mathrm{mg} / \mathrm{dL}, 1$-hour $180 \mathrm{mg} / \mathrm{dL}, 2$-hour $155 \mathrm{mg} / \mathrm{dL}$, 3-hour $140 \mathrm{mg} / \mathrm{dL}(8)$.

Patient demographic characteristics such as maternal age, gravity, parity, gestational week, BMI, and laboratory parameters including hepatic enzymes, fasting and postprandial TBA levels were recorded. Gestational age was determined according to The International Society of Ultrasound in Obstetrics and Gynecology (ISUOG) practice guidelines for the first trimester ultrasound (9). Gestational age at delivery, mean birthweight (BW), Neonatal Intensive Care Unit (NICU) admission requirement, meconium-stained amniotic fluid and Apgar score at the $5^{\text {th }}$ minutes were recorded to evaluate the perinatal outcome.

Statistical Package for Social Sciences, Windows version 15.0 (SPSS, Chicago, IL, USA) was used to analyze the variables. The results of descriptive statistics were expressed as mean \pm standard deviation and median (minimum-maximum) for numerical variables. The normality of the data distribution was assessed with the Kolmogorov-Smirnov test. Parametric data were examined with independent two-sample $t$ test and non-parametric data were compared using the Mann Whitney$\mathrm{U}$ test. Categorical variables were expressed as number (percentage). Percentages were compared with the chi-square test. Associations between variables were evaluated using Pearson's correlation analyses. Logistic regression analyses were performed to calculate the odds ratios (OR) with a 95\% confidence interval. A $p$ value $<0.05$ indicates statistically significant differences.

\section{Results}

A total of 160 pregnant women (80 diagnosed with ICP and 80 patients as controls) were included in our study. Mean age was 27.4 \pm 5.1 , mean BMI was $28.1 \pm 3.7$, mean value of 50g GCT was $117.5 \pm 29.6$ and the percentage of GDM was $8.75 \%$ in our study population.

There were no statistically significant differences in terms of age, gravidity, parity, and BMI between two groups of pregnant women. Hepatic enzyme levels including mean alanine aminotransferase (ALT) and aspartate aminotransferase (AST) were significantly higher in the ICP group than the control group. Gestational age at birth and birthweight were lower in the ICP group than the control group ( $p=0.001$ and $p=0.016$, respectively). Demographic data and laboratory test results of the two groups are presented in table I.

Also no significant differences were found regarding age, gravidity, parity and BMI between two ICP groups divided as mild and severe. Mean fasting and postprandial TBA levels 
were significantly higher in the severe group than the mild group. Gestational age at birth and birthweight were lower in pregnant women with severe disease than those with the mild disease ( $p=0.001, p=0.016$, respectively). Demographic information and laboratory parameters of the two ICP groups are shown in table II.

In this study population, the result of mean 50-g GCT was higher in pregnant women with ICP $(128.7 \pm 28.2)$ compared to the healthy pregnant control group $(106.6 \pm 27.0)(p=0.0001)$. The mean 50-g GCT value was slightly higher in women with severe disease than those with mild disease but the difference did not reach the significance level $(132.7 \pm 30.1,125.1 \pm 26.5$, respectively; $p=0.26$ ) (Figure 1). Although the difference was not significant, GDM rates were higher in women with ICP compared to the healthy pregnant group $(11.25 \%, 6.25 \%$, respectively; $p=0.187$ ), (Table I). In addition, GDM rates were similar in pregnant women with mild and severe disease $(11.1 \%, 11.4 \%$, respectively; $p=0.31)$ (Table II).

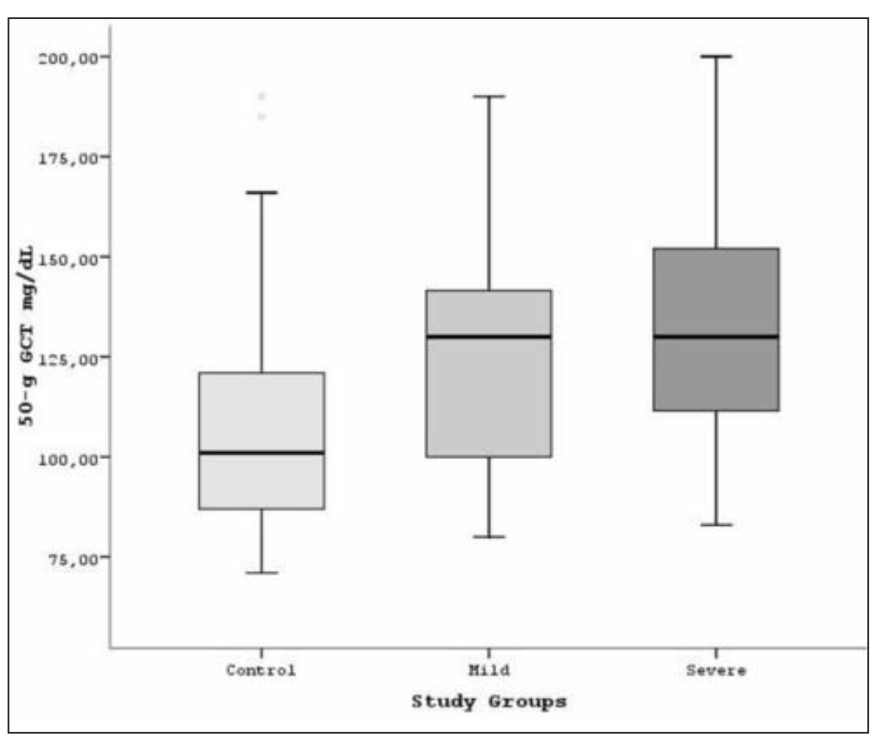

Figure 1: Comparison of Mean $50 \mathrm{~g}$ GCT Levels between Study Groups.

Table I: Comparison of demographic and laboratory findings of intrahepatic cholestasis of pregnancy and control group

\begin{tabular}{lccc}
\hline Variables & ICP Group $(\mathrm{n}=80)$ & Control Group $(\mathrm{n}=80)$ & $p$ value \\
\hline Age (years) & $27.6 \pm 5.0$ & $27.2 \pm 5.2$ & 0.62 \\
Gravidity & $2.0 \pm 1.2$ & $2.3 \pm 1.5$ & 0.14 \\
Parity & $0.5 \pm 0.7$ & $0.6 \pm 1.0$ & 0.44 \\
BMI (kg/m ${ }^{2}$ ) & $27.9 \pm 3.8$ & $28.5 \pm 3.4$ & 0.48 \\
ALT (U/L) & $138.0 \pm 115.7$ & $14.2 \pm 7.7$ & 0.0001 \\
AST (U/l) & $94.4 \pm 74.7$ & $18.3 \pm 6.5$ & 0.0003 \\
Fasting TTBA (mmol/L) & $40.2 \pm 42.4$ & - & - \\
Postprandial TTBA (mmol/L) & $42.5 \pm 51.9$ & - & - \\
Gestational age at birth (week) & $36.3 \pm 1.7$ & $38.1 \pm 1.9$ & 0.0004 \\
Neonatal Birthweight (g) & $2891.3 \pm 473.7$ & $3141.4 \pm 481.7$ & 0.001 \\
FPG (mg/dL) & $82.6 \pm 8.2$ & $78.5 \pm 11.0$ & 0.06 \\
50-g GCT (mg/dL) & $128.7 \pm 28.2$ & $106.6 \pm 27.0$ & 0.0001 \\
Incidence of GDM, $n(\%)$ & $9(11.25 \%)$ & $5(6.25 \%)$ & 0.187 \\
\hline
\end{tabular}

Data expressed as mean $\pm S D$. ICP: Intrahepatic cholestasis of pregnancy, BMI: Body mass index, ALT: Alanine aminotransferase, AST: Aspartate aminotransferase, TTBA: Total TBA, FPG: Fasting plasma glucose, GCT: Glucose challenge test, GDM: Gestational diabetes mellitus

Table II: Comparison of demographic and laboratory findings of mild and severe intrahepatic cholestasis of pregnancy group

\begin{tabular}{lccc}
\hline Variables & Mild ICP Group $(\mathrm{n}=45)$ & Severe ICP Group $(\mathrm{n}=35)$ & $p$ value \\
\hline Age(years) & $27.6 \pm 4.4$ & $27.5 \pm 5.6$ & 0.99 \\
Gravidity & $2.1 \pm 1.2$ & $1.8 \pm 1.2$ & 0.26 \\
Parity & $0.6 \pm 0.8$ & $0.4 \pm 0.7$ & 0.18 \\
BMI (kg/m ${ }^{2}$ ) & $28.5 \pm 3.5$ & $27.9 \pm 3.8$ & 0.48 \\
ALT (U/L) & $115.5 \pm 103.0$ & $166.3 \pm 125.8$ & 0.05 \\
AST (U/L) & $82.2 \pm 67.5$ & $109.7 \pm 81.3$ & 0.10 \\
Fasting TBA (mmol/L) & $22.3 \pm 9.1$ & $67.9 \pm 69.9$ & 0.0005 \\
Postprandial TBA (mmol/L) & $21.3 \pm 8.9$ & $65.4 \pm 55.02$ & 0.0001 \\
Gestational age at birth (week) & $36.9 \pm 1.2$ & $35.6 \pm 2.0$ & 0.001 \\
Neonatal Birthweight (g) & $2745.4 \pm 482.0$ & $3008.7 \pm 438.1$ & 0.016 \\
FPG (mg/dL) & $78.5 \pm 11.0$ & $82.6 \pm 8.2$ & 0.06 \\
50-g GCT (mg/dL) & $125.5 \pm 26.5$ & $132.7 \pm 30.1$ & 0.26 \\
Incidence of GDM, $\mathrm{n}(\%)$ & $5(11.1 \%)$ & $4(11.4 \%)$ & 0.31 \\
\hline
\end{tabular}

Data expressed as mean \pm SD. BMI: Body mass index; ALT: Alanine aminotransferase, AST: Aspartate aminotransferase, TTBA: Total TBA, FPG: Fasting plasma glucose, GCT: Glucose challenge test, GDM: Gestational diabetes mellitus, ICP: Intrahepatic cholestasis of pregnancy 
To evaluate a possible association between 50 -g GCT value and TBA, ALT and AST levels in pregnant women with ICP, bivariate correlation analyses were used. In subgroup analyses of women with ICP, there was not any association between the 50-g GCT value and fasting TBA level ( $r=0.057$, $p=0.62)$ or postprandial TBA level $(r=0.02, \mathrm{p}=0.82)$. There was also no correlation between 50 -g GCT value and ALT level $(r=0.05, p=0.63)$ or AST level $(r=0.07, p=0.51)$ in ICP pregnant women.

In the logistic regression model, although statistically not significant, ICP disease increased the risk of GDM 1.9- fold (95\% CI: $0.71-3.31 ; p=0.24$ ). Also the risk of positive screening rate in 50-g GCT increased 3.6-fold for ICP pregnant women (95\% CI: 1.6-8; $p=0.01)$.

\section{Discussion}

In the current study, we found that the mean 50-g GCT value was significantly higher in pregnant women with ICP than healthy pregnant controls. Although the percent of GDM diagnosis was higher in ICP pregnant women than controls and also in severe disease versus mild disease, the differences did not reach statistical significance. We found that while ICP disease insignificantly increases the risk of GDM by 1.9 fold, it significantly increased the risk of positive GDM screening by 3.6 fold.

The GDM definition is based on the glucose intolerance that is not overt before pregnancy and diagnosed second or third trimester during pregnancy (10) and the disease has important short term and long term effects on maternal and fetal well-being $(11,12)$. GDM increases risks of perinatal complications and constitutes a risk for fetal macrosomia, shoulder dystocia and neonatal hypoglycemia (13). It is also demonstrated that women with a history of GDM have a high risk of developing dyslipidemia, obesity and insulin resistance as part of metabolic syndrome later in life $(14,15)$. Due to the high risk for both the mother and fetus, its diagnosis and treatment during pregnancy should be managed accurately.

Different from our results, previous studies have reported an association between ICP and GDM $(3,7)$. Wikström et al. (7) showed that pregnant women diagnosed with ICP tend to have GDM and large for gestational age infants at birth. More recently, Martineau et al. found that the incidence of GDM was higher in pregnancies with ICP and that this association increased more after the onset of cholestasis (3). We found that pregnant women with ICP had higher mean 50-g GCT values than the controls which is similar to the literature, however GDM rates were higher in pregnant women with ICP but this difference did not reach statistical significance in this study. The association between obesity and GDM is well known (16), however, in our study BMI was similar between the two groups.

BAs play an important role in dietary lipid absorption and cholesterol homeostasis. Also in recent animal modality studies it was demonstrated that BAs could take part in the regulation of glucose homeostasis by activating the cell surface and nuclear receptors as hormonal signaling molecules (17). As a primary BA receptor, FXR has a regulatory role in both glucose and cholesterol homeostasis (18). Gao et al. demonstrated that women with GDM have a significant increase in 8 BA species compared to the controls (19). Increased serum BA levels are a distinctive feature of ICP and it can be thought that GDM and ICP could share the same pathogenesis through BAs as the cornerstone. Nevertheless, the relationship between serum BA concentrations and metabolic syndrome or type 2 DM could not yet be demonstrated in humans (20). Although the diagnosis rate of GDM was found to be higher in the severe ICP group, in which the measurement of serum BA level was greater than $40 \mathrm{mmol}$ compared to the mild group, we could not demonstrate a significant association between serum BA level and mean 50-g GCT value. To the best of our knowledge, our study is the first one in the literature that evaluated the relationship between BA levels and 50-g GCT value.

In many studies, it was demonstrated that ICP has an association with an increased risk of poor fetal and neonatal outcomes such as preterm delivery, fetal stress during labor, meconium-stained amniotic fluid, and intrauterine fetal death $(5,21)$. Although severe ICP disease with serum TBA level $>40 \mathrm{mmoL} / 1$ has been shown to be associated with an increased risk of meconium-stained amniotic fluid, low APGAR scores and fetal intrauterine death in recent studies $(5,21)$, optimal time for delivery and best predictors of the neonatal outcome before delivery are still unknown. Because of the increased rates of fetal death and postnatal complications, delivery before 37 weeks of pregnancy is usually recommended for these pregnancies (22). It's logical to found a statistically significant difference between ICP and healthy control group in terms of BW and gestational age at birth due to our policy of early delivery of ICP patients according to the recommendations in the literature.

\section{Conclusion}

In conclusion, our study showed that pregnant women with ICP had a higher incidence of GDM. But this difference did not reach statistical significance. Also, we could not find an association between TBA levels and serum 50-g GCT. Our results are not similar to the literature. It may be due to our small sample size. So, further and well-designed studies with larger sample sizes are necessary to determine the relationship between GDM and ICP and also the function of BA in the pathogenesis of GDM disease.

: Acknowledgement: None

Conflict of Interest: No conflict of interest

Funding Source: None 
Author Contributions: GGT: Concept, Writer, Design, Data processing, Analysis and interpretation. ZVY: Data processing, Analysis and interpretation, Design, Statistical analysis. YO: Data collection, Processing, Analysis, and interpretation. KY: Data collection, Processing, Analysis, and interpretation. CYS: Supervision,Statistical analysis,Design.DU: Supervision, Concept

\section{References}

1. Geenes V, Williamson C. Intrahepatic cholestasis of pregnancy. World J Gastroenterol. 2009;15(17):2049-66.

2. Williamson C, Geenes V. Intrahepatic cholestasis of pregnancy. Obstet Gynecol. 2014;124(1):120-33.

3. Martineau M, Raker C, Powrie R, Williamson C. Intrahepatic cholestasis of pregnancy is associated with an increased risk of gestational diabetes. Eur J Obstet Gynecol Reprod Biol. 2014;176:80-5.

4. Kirbas A, Biberoglu E, Daglar K, İskender C, Erkaya S, Dede H, et al. Neutrophil-to-lymphocyte ratio as a diagnostic marker of intrahepatic cholestasis of pregnancy. Eur J Obstet Gynecol Reprod Biol. 2014;180:12-5.

5. Glantz A, Marschall HU, Mattsson LA. Intrahepatic cholestasis of pregnancy: relationships between bile acid levels and fetal complication rates. Hepatology. 2004; 40(2):467-74

6. Brouwers L, Koster MP, Page-Christiaens GC, Kemperman H, Boon J, Evers IM, et al. Intrahepatic cholestasis of pregnancy: maternal and fetal outcomes associated with elevated bile acid levels. Am J Obstet Gynecol. 2015;212(1):100.e1-7.

7. Wikström Shemer E, Marschall H, Ludvigsson JF, Stephansson O. Intrahepatic cholestasis of pregnancy and associated adverse pregnancy and fetal outcomes: a 12year population based cohort study. BJOG. 2013; 120(6):717-23.

8. Obstetricians ACo, Gynecologists. Gestational Diabetes Mellitus. Practice Bulletin. No.190. Obstet Gynecol. 2018;131(2):49-64.

9. Salomon LJ, Alfirevic Z, Bilardo CM, Chalouhi GE, Ghi T, Kagan KO, et al. ISUOG Practice Guidelines: performance of first-trimester fetal ultrasound scan. Ultras Obstet Gynecol. 2013;41(1):102-13.

10. American Diabetes Association. 2. Classification and diagnosis of diabetes: standards of medical care in diabetes2018. Diabetes Care. 2018;41(Suppl 1):S13-S27.

11. Catalano PM, McIntyre HD, Cruickshank JK, McCance
DR, Dyer AR, Metzger BE, et al. The hyperglycemia and adverse pregnancy outcome study: associations of GDM and obesity with pregnancy outcomes. Diabetes Care. 2012;35(4):780-6.

12. Crowther CA, Hiller JE, Moss JR, McPhee AJ, Jeffries WS, Robinson JS. Effect of treatment of gestational diabetes mellitus on pregnancy outcomes. N Engl J Med. 2005;352 (24):2477-86.

13. Yogev Y, Visser GH, editors. Obesity, gestational diabetes and pregnancy outcome. Semin Fetal Neonatal Med. 2009;14(2):77-84.

14. Bellamy L, Casas J-P, Hingorani AD, Williams D. Type 2 diabetes mellitus after gestational diabetes: a systematic review and meta-analysis. Lancet. 2009;373(9677):1773-9.

15. Gunderson EP, Hurston SR, Dewey KG, Faith MS, Charvat-Aguilar N, Khoury VC, et al. The study of women, infant feeding and type 2 diabetes after GDM pregnancy and growth of their offspring (SWIFT Offspring study): prospective design, methodology and baseline characteristics. BMC Preg Child. 2015;15(1):150.

16. Abrams B, Parker J. Overweight and pregnancy complications. Int J Obes 1988;12(4):293-303.

17. Prawitt J, Caron S, Staels B. Bile acid metabolism and the pathogenesis of type 2 diabetes. Curr Diab Rep. 2011;11(3):160-6.

18. Cariou B, Staels B. FXR: a promising target for the metabolic syndrome? Trend Pharmacol Sci. 2007;28(5):23643.

19. Gao J, Xu B, Zhang X, Cui Y, Deng L, Shi Z, et al. Association between serum bile acid profiles and gestational diabetes mellitus: a targeted metabolomics study. Clin Chim Acta. 2016;459:63-72.

20. Steiner C, Othman A, Saely CH, Rein P, Drexel H, von Eckardstein A, et al. Bile acid metabolites in serum: intraindividual variation and associations with coronary heart disease, metabolic syndrome and diabetes mellitus. PLoS One. 2011;6(11):e25006.

21. Geenes V, Chappell LC, Seed PT, Steer PJ, Knight M, Williamson C. Association of severe intrahepatic cholestasis of pregnancy with adverse pregnancy outcomes: a prospective population based case control study. Hepatology. 2014;59(4):1482-91.

22. Lo JO, Shaffer BL, Allen AJ, Little SE, Cheng YW, Caughey AB. Intrahepatic cholestasis of pregnancy and timing of delivery. J Matern Fetal Neonatal Med. 2015;28(18): 2254-8. 